\title{
Novel techniques for weak alignment of proteins in solution using chemical tags coordinating lanthanide ions
}

\author{
Takahisa Ikegami $^{\mathrm{a}, * *}$, Laurent Verdier ${ }^{\mathrm{a}, * *}$, Peyman Sakhaii ${ }^{\mathrm{a}, * *}$, Susanne Grimme ${ }^{\mathrm{b}}$, Barbara \\ Pescatore $^{\mathrm{b}}$, Krishna Saxena ${ }^{\mathrm{b}}$, Klaus M. Fiebig ${ }^{\mathrm{c}}$ \& Christian Griesinger ${ }^{\mathrm{a}, *}$ \\ ${ }^{a}$ Max-Planck Institute for Biophysical Chemistry, Department of NMR Based Structural Biology, University of \\ Frankfurt am Faßberg 11, D-37077 Göttingen, Germany \\ ${ }^{\mathrm{b}}$ Institute for Organic Chemistry, Marie-Curie-Str. 11, D-60439 Frankfurt am Main, Germany \\ ${ }^{\mathrm{c}}$ Affinium Pharmaceutical, 100 University Avenue, Toronto, Ontario M5J 1V6, Canada
}

Received 24 December 2003; Accepted 3 March 2004

Key words: dipolar coupling, disulfide bond, lanthanide ion, molecular alignment, NMR

\begin{abstract}
A molecule with an anisotropic magnetic susceptibility is spontaneously aligned in a static magnetic field. Alignment of such a molecule yields residual dipolar couplings and pseudocontact shifts. Lanthanide ions have recently been successfully used to provide an anisotropic magnetic susceptibility in target molecules either by replacing a calcium ion with a lanthanide ion in calcium-binding proteins or by attaching an EDTA derivative to a cysteine residue via a disulfide bond. Here we describe a novel enantiomerically pure EDTA derived tag that aligns stronger due to its shorter linker and does not suffer from stereochemical diversity upon lanthanide complexation. We observed residual ${ }^{15} \mathrm{~N},{ }^{1} \mathrm{H}$-dipolar couplings of up to $8 \mathrm{~Hz}$ at $800 \mathrm{MHz}$ induced by a single alignment tensor from this tag.
\end{abstract}

\section{Introduction}

The usage of dipolar couplings in order to obtain more accurate structures as well as information on dynamics has been proven over the past years (Tolman et al., 1995; Tjandra and Bax, 1997; Clore and Gronenborn, 1998). Since diamagnetic molecules do not show enough alignment in a strong magnetic field, a large array of external alignment media have been introduced. By contrast, paramagnetic molecules do not require external alignment media (Tolman et al., 1995). In addition to dipolar couplings, they also exhibit paramagnetic relaxation as well as pseudocontact shifts that can be used as restraints for structure calculations. Diamagnetic molecules can be converted into paramagnetic molecules by attaching a paramagnetic tag consisting of a small organic molecule that

\footnotetext{
*To whom correspondence should be addressed. E-mail: cigr@nmr.mpibpc.mpg.de

**These authors have equally contributed to this work.
}

specifically binds a lanthanide. Such a molecule is EDTA, which has to be suitably functionalized to allow for tagging of the protein. EDTA based tags benefit from their sub-picomolar dissociation constants for lanthanides that makes them robust against unfolding conditions at neutral $\mathrm{pH}$. Protein/ligand complexes can be more easily studied since the ligand does not orient while not bound to the tagged protein. This will be especially beneficial when studying hydrophobic ligands that interact with the aligning medium (e.g. phage, bicelles or gel) in competition with the target protein and adopt broad lines. The last advantage is in the study of dynamics since alignment tensors can be determined independently from pseudocontact shifts as well as the dipolar couplings. If there is dynamics that would modulate the alignment tensor in external alignment media, this can be detected clearly from paramagnetic tagging. In addition, it has been shown that different lanthanide ions induce different alignment tensors when bound to the same protein binding 
pocket (Bertini et al., 2001a). This behavior can also be expected for lanthanides bound to EDTA derived tags such that analysis of dynamics using at least five different alignment media will be feasible.

The fact that a tag will move with respect to the protein will lead to an averaged alignment tensor for the protein. This is not a problem for the interpretation of dipolar couplings while the motional averaging of the tag needs to be taken into account when pseudocontact shifts are interpreted.

The idea of using lanthanide ions for inducing anisotropic susceptibilities in diamagnetic molecules is not novel. Lanthanides have recently been used to provide a target molecule with an anisotropic magnetic susceptibility partly because they do not exhibit paramagnetic relaxation effects as severe as other transition metal ions (Burns and LaMar, 1982; Bertini et al., 2001b,c). Examples include the incorporation of europium into quadruplex DNA (Beger et al., 1998), replacement of a calcium ion with a lanthanide ion in calcium-binding proteins (Bertini et al., 2001d; Contreras et al., 1999), attaching an EF-hand motif (Ma and Opella, 2000) or complete calmodulin (Feeney et al., 2001) that coordinates lanthanide ions to the protein under investigation. Alternatively, an optimized short peptide that binds lanthanides can be attached to the $\mathrm{C}$ - or N-terminus of the protein under investigation (Wöhnert et al., 2003). Lanthanide ions can also be used to change the alignment orientation of a liquid crystal (DHPC/DMPC) when these metals are added to a bicellar system in a form chelated by DMPE-DTPA (1,2-dimyristoyl-sn-glycero-3phosphoethanolaminediethylenetriaminepentaacetate) (Prosser et al., 1998). Although not utilizing lanthanide ions, some techniques to attach other metal ions, or groups having an unpaired electron, to proteins have been developed. For example, binding of $\mathrm{Co}^{2+}$ to a zinc finger-fused protein (Gaponenko et al., 2000, 2002) introduced an anisotropic paramagnetic susceptibility, providing orientational information based on dipolar coupling constants. Similarly, $\mathrm{Co}^{2+}$ was successfully used for DNA structure elucidation (Gochin, 2000). Furthermore, ATCUN, a threeamino acid motif that binds $\mathrm{Cu}^{2+}$ was fused to the $\mathrm{N}$-terminal end of a protein (Donaldson et al., 2001) and spin labels were bound to cysteine residues (Battiste and Wagner, 2000) to obtain distance information from paramagnetic relaxation enhancement. Other interesting ideas of applying trapped metal ions to biological systems include conjugating iron chelates, such as iron ( $S$ )-1-( $p$-bromoacetamidobenzyl) EDTA
(Datwyler and Meares, 2000), (EDTA-2-aminoethyl) 2-pyridyl disulfide (Ermacora et al., 1992), and $S$-(2pyridylthio) cysteaminyl-EDTA (Elbright, 1992), to cysteine residues in proteins in order to allow intra- or inter-molecular cleavage to occur. A localized cleavage would indicate a protein-protein interaction site or a protein conformational change.

Here, we present another method of attaching metal ions, especially lanthanide ions, to proteins to align them in a static magnetic field. We chemically synthesized three modified EDTA compounds that can coordinate a lanthanide ion and bind to a cysteine residue through a disulfide bond. While the first compound has been recently successfully used with $\mathrm{Co}^{2+}$ (Gaponenko et al., 2000, 2002; Dvoretsky et al., 2002), in our hands the $\mathrm{Dy}^{3+}$ bound form of a tagged protein exhibited dipolar couplings with a maximum of only $1.5 \mathrm{~Hz}$ and led to a duplication of signals. The second and third compounds (mutual diastereomers) have, however, a shorter linker and defined stereochemistry. They therefore provide large dipolar couplings and a unique set of signals.

\section{Experimental procedures}

\section{The three EDTA-derived compounds}

The EDTA-compound 1 S-Methanesulfonyl-Lcysteaminyl-1-carbonic-acid-ethylenediamine- $\mathrm{N}, \mathrm{N}, \mathrm{N}^{\prime}$, $\mathrm{N}^{\prime}$-tetraacetic acid (molecular weight of $429.469 \mathrm{Da}$ ) was synthesized as will be published elsewhere (Figure 1a). It can be purchased from Toronto Research chemicals (M258835) which made it an obvious choice to introduce metal chelators. The compound is achiral. However, upon complexation with a lanthanide, the threefold asymmetrically substituted nitrogen becomes chiral, yielding a mixture of enantiomers. Covalent linking to a chiral protein then produces two diastereomers which are expected to yield different spectra (vide infra).

The EDTA-carbonic acid compound $\mathbf{3}$ is S-Methanesulfonyl-L-cysteaminyl-1R-carbonic-acid-ethylenediamine-N,N, $\mathrm{N}^{\prime}, \mathrm{N}^{\prime}$-tetraacetic acid (EDTA-CAMTS) and has a molecular weight of 517 Da (Figure 1b). It was synthesized in five steps by condensation of racemic 2,3-bis[di(tert-butoxycarbonylmethyl) amino]propionic acid (Arya and Garyepy, 1991) with (L)-S-mesylcysteine (Weidner and Block, 1972) which were prepared by modification of known methods. The diastereomeric mixture was separated by 
a)

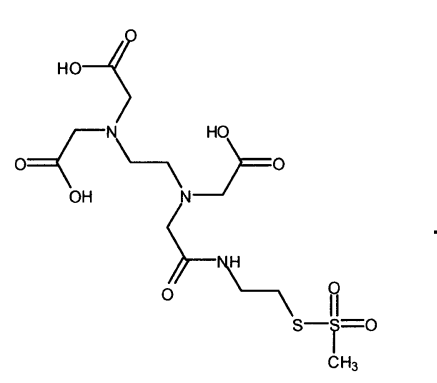

MTS-EDTA: 1<smiles>[R]C(=O)C(CS)NC</smiles>

TF-S100C

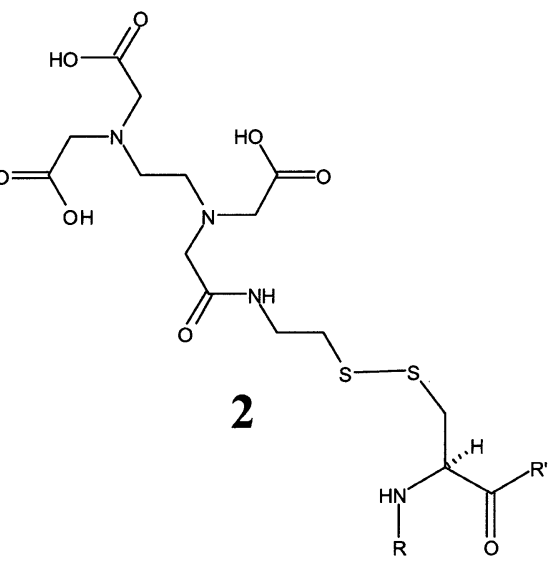

b)

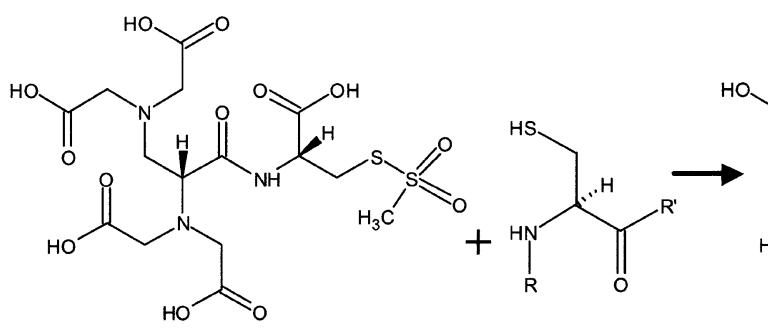

\section{MTS-EDTA-CA: 3 TF-S100C}<smiles>CS(=O)(=O)SC[C@H](NC(=O)C(CN(CC(=O)O)CC(=O)O)N(CC(=O)O)CC(=O)O)C(=O)O</smiles><smiles>[R]N[C@H](CS)C([R])=O</smiles>

\section{4}<smiles>[120In]</smiles>

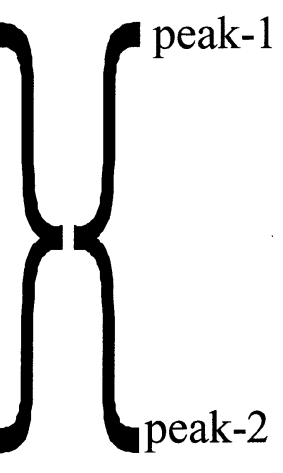

Figure 1. (a) The tagging reactions between MTS-EDTA ([S-Methanesulfonylcysteaminyl]-EDTA) 1 and the Cys100 on Trigger-factor forming 2. (b) Same for MTS-EDTA-carbonic acid $\mathbf{3}$ and 1-epi-3 forming $\mathbf{4}$ and 1-epi-4. Since we did not have the assignment of the diastereomers, we distinguished them by the different retention times in HPLC as peak-1 and peak-2. (c) EDTA-Cysteine 5 and EDTA- $\alpha$-Methylbenzylamide $\mathbf{6}$.

HPLC after final deprotection to give (D,L) and (L,L) compounds. A full account of the synthesis will be given elsewhere. The compound can be obtained from the authors (cigr@nmr.mpibpc.mpg.de). 3 is chiral and four stereoisomers exist. By contrast to compound 1, complexation with the metal ion does not induce further stereochemistry, since both nitrogens are symmetrically substituted. Compound $\mathbf{3}$ and all its stereoisomers are therefore enantiomerically pure even after complexation with lanthanide. In this work both diastereomers originating from L-cysteine were investigated, namely 3 and 1-epi-3.
Sample preparation of TF-S100C-EDTA-lanthanide and TF-S100C-EDTA-carbonic-acid-lanthanide compound

A cysteine residue was introduced in Trigger-Factor (molecular weight (M.W.) $=13851.9$ Da, extinction coefficient at $280 \mathrm{~nm}: 3840 \mathrm{M}^{-1} \mathrm{~cm}^{-1}$ ) by a site-directed mutagenesis at the position of Ser-100, which resides on the surface of the protein and is well exposed to solvent (solvent accessibility of Ser$100=40 \%$ ). The mutant, TF-S100C, was purified in the same way as the wild type (Vogtherr et al., 2002) with one exception. In all buffer solutions used for the purification, $5 \mathrm{mM}$ Dithiothreitol (DTT) was 

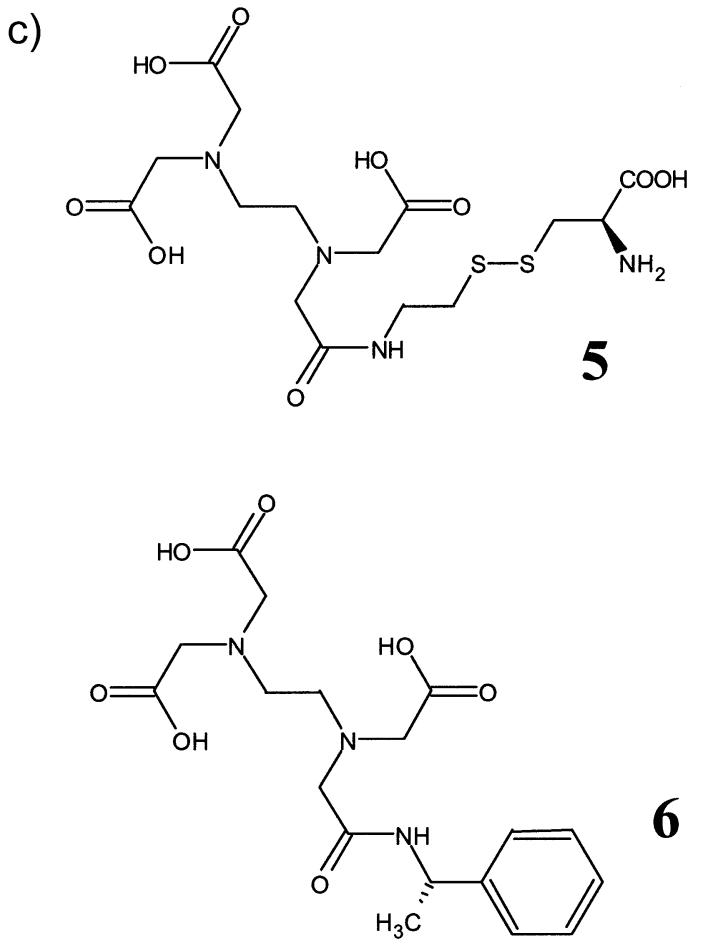

Figure 1. Continued.

added to prevent nonspecific intermolecular disulfide bond formation. A solution containing TF-S100C was finally applied to a Mono-S (Pharmacia) cation exchange chromatography. The column had been equilibrated in advance with a buffer containing $50 \mathrm{mM}$ sodium phosphate (pH 6.5) and 5 mM DTT. The protein was eluted by application of a linear gradient of the $\mathrm{NaCl}$ concentration from 0 to $1000 \mathrm{mM}$. A 44 fold excess of compound 1 was added to the eluted fraction containing concentrated TF-S100C. After $24 \mathrm{~h}$ on ice, the tagging reaction was stopped by replacing the solvent with a solution containing $50 \mathrm{mM}$ MOPS (3-Morpholinopropanesulfonic acid) buffer ( $\mathrm{pH} 7.0$ ), $50 \mathrm{mM} \mathrm{NaCl}$, and no reducing agent by an ultrafiltration method (Millipore, Centricon YM-3, the molecular cut was $3000 \mathrm{Da}$ ) and $5 \mathrm{mg}$ (per 11 culture) of the tagged TF-S100C compound 2 was isolated (Figure 1a).

The coupling reactions of TF-S100C with compound $\mathbf{3}$ and 1-epi-3 were performed after removing DTT by centricon filtration and using only a threefold excess. The tagged compounds 4 and 1-epi-4 were isolated with $90 \%$ yield (Figure $1 \mathrm{~b}$ ).

Lanthanum chloride $\left(\mathrm{LaCl}_{3}\right)$ and Dysprosium chloride $\left(\mathrm{DyCl}_{3}\right)$ were weighed and dissolved in the same MOPS buffer to the concentration of $100 \mathrm{mM}$. Since the $\mathrm{pH}$ values of the metal solutions were only 6.5 , they were adjusted to 7.0 with a small amount of $1 \mathrm{~N} \mathrm{NaOH}$. Each metal ion was added into the protein solution with four times the molarity of the protein. After sitting overnight, the excess metal ions were washed out by replacing the solvent four times with the MOPS buffer containing $10 \% \quad \mathrm{D}_{2} \mathrm{O}$ using Centricon-3, and the solution was concentrated at the same time. After an excess of lanthanide was added, the solution was always washed with the buffer in order to remove excess lanthanide. A solution of $250 \mu \mathrm{l}$ was sealed in a Shigemi NMR sample tube of $5 \mathrm{~mm}$ diameter with an adaptor-piston inserted upward (BMS-3) for NMR measurements.

\section{Preparation of Cys-EDTA-metal and $\alpha$-Methylbenzylamide-EDTA- metal compound}

The preparation of the compound is described elsewhere (P. Sakhaii et al., submitted) (Figure 1c).

\section{Binding strength between the EDTA compound and the lanthanides}

The metal/EDTA-compound complexes were prepared by adding an excess of lanthanide and then removing it by washing. The efficiency of the washing was controlled in the following way: The line widths of most of the resonances in the ${ }^{1} \mathrm{H},{ }^{15} \mathrm{~N}$ HSQC were similar to those of the diamagnetic compound and there were no cross peaks indicating tag that was not chelated by a metal. The fact that this procedure works indicates that the modified EDTA compounds have a very high association constant with lanthanides.

\section{Measurement of scalar and dipolar coupling constants}

For compound 2, dipolar coupling constants were not evaluated due to the doubling of resonances originating from stereoisomers as described below (Figure 2). For compounds 4 and 1-epi-4, $\omega_{1}$ coupled HSQC spectra were recorded since the couplings turned out to be large enough at $800 \mathrm{MHz}$ to be directly read off from the spectrum. $\omega_{1}$-traces were added up and the coupling constants were extracted by superimposition of the high-field on the low-field submultiplet line or vice versa. The errors were determined by adding noise to the traces derived from peak-free regions of the spectrum. 
(a)

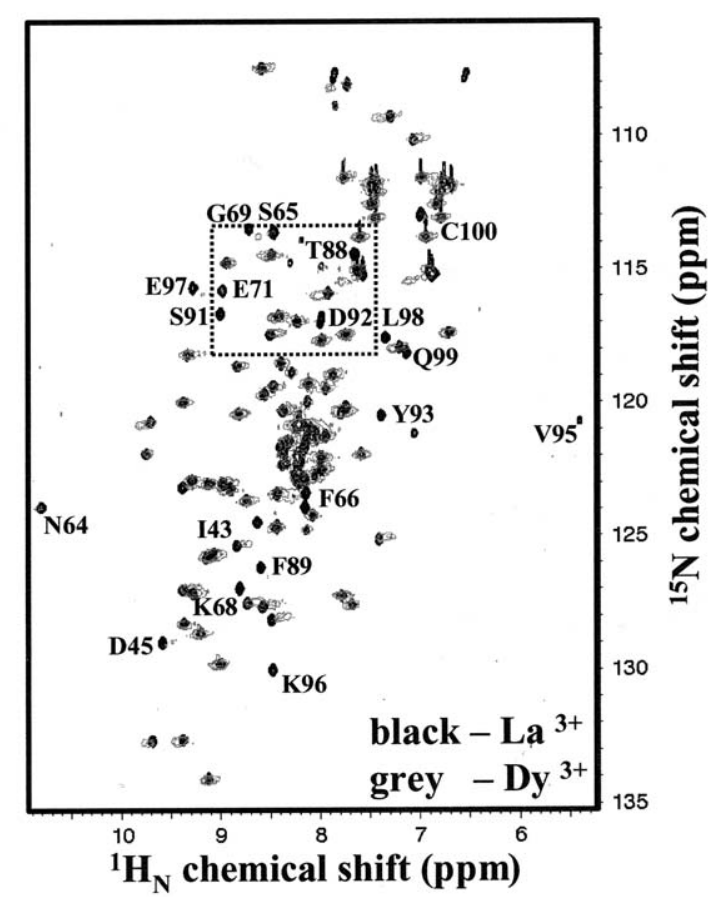

(b)

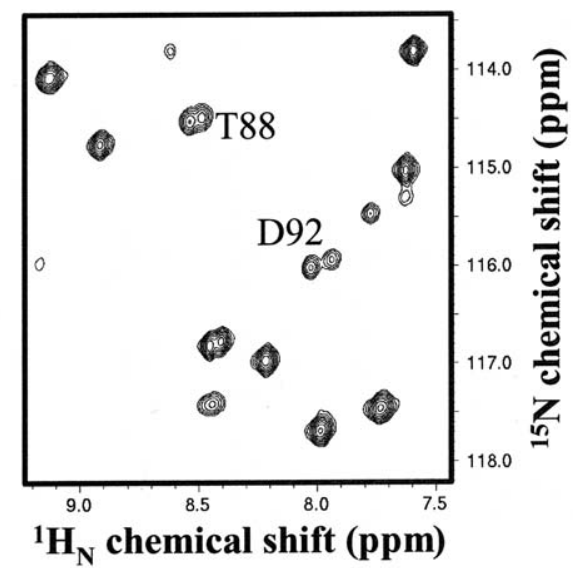

Figure 2. (a) Overlay of the two-dimensional ${ }^{1} \mathrm{H}_{-}{ }^{15} \mathrm{~N}-\mathrm{HSQC}$ spectra of TF-S100C-EDTA- Dy ${ }^{3+}$ (grey) and TF-S100C-EDTA- La ${ }^{3+}$ (black). Peaks of the resonances are labeled that were broadened owing to paramagnetic relaxation in the presence of four equivalent amount of Dy ${ }^{3+}$ compared to the amount of the proteins. (b) A magnified part of the HSQC spectrum, corresponding to the region boxed by the dashed lines in (a), in the presence of $\mathrm{Dy}^{3+}$. Doublet forms were observed in some peaks. Both spectra were acquired at $303 \mathrm{~K}$ with a Bruker DRX600 spectrometer with a pulse sequence containing water-flip-back and WATERGATE methods.

\section{Results}

Preparation of TF-S100C-EDTA-Dy and TF-S100CEDTA-carbonic-acid-Dy

We successfully prepared TF-S100C-EDTA 2 (Figure 1a) as evidenced by a MALDI mass spectrum (not shown), which showed an increase in the molecular weight of the protein after the reaction by the expected 350.0 Da. The tagging to the proteins was complete since no mass peak of free TF-S100C was observed. Successful metal chelation of the tagged protein with lanthanides, as well as the stability of the complex, was evidenced by MALDI mass spectrometry conducted in a $50 \mathrm{mM}$ MOPS buffer $(\mathrm{pH}$ 7.0) for the protein and metal solutions. A phosphate buffer could not be used because of precipitation of a metal-phosphate complex. We maintained the $\mathrm{pH}$ value at 7.0 for the final solution because the metal complexation is $\mathrm{pH}$ dependent, due to the relevant carboxyl groups being discharged through protonation.
Similarly, EDTA-Cys, compound $\mathbf{5}$, and EDTA- $\alpha$ Methylbenzylamide, compound $\mathbf{6}$, were obtained with approximately $100 \%$ yield.

TF-S100C-EDTA-carbonic acid, 4, and its 1epimer, 1-epi-4, were prepared and analyzed by ESImass spectrometry. According to the mass spectrum the reaction was complete in both cases. We obtained two peaks, one at $518 \mathrm{Da}$ corresponding to the excess of compound $\mathbf{3}$ in the reaction mixture and the other one at $10802 \mathrm{Da}$ fitting perfectly to the sum of $438 \mathrm{Da}$ and $10364 \mathrm{Da}$ (trigger factor 19-113) present in compounds 4 and 1-epi-4. The assignments to the two diastereomers $\mathbf{3}$ and 1 -epi- $\mathbf{3}$ could not yet be done but the diastereomers were characterized from the retention times in HPLC. The reagent with the shorter retention time is called 'peak 1' and the one with the longer retention time 'peak 2'. 
(a)

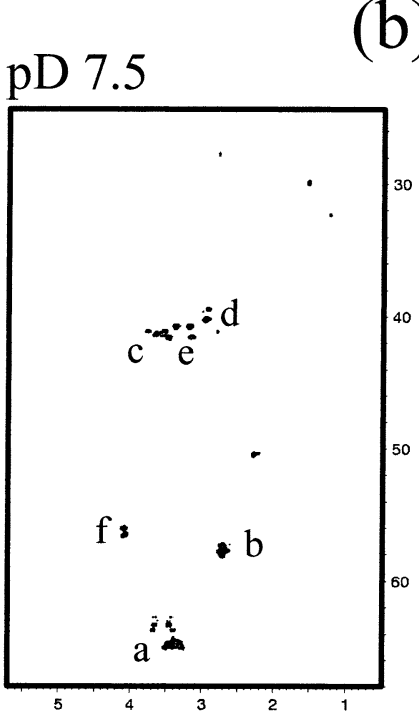

${ }^{1} \mathrm{H}$ chemical shift (ppm)

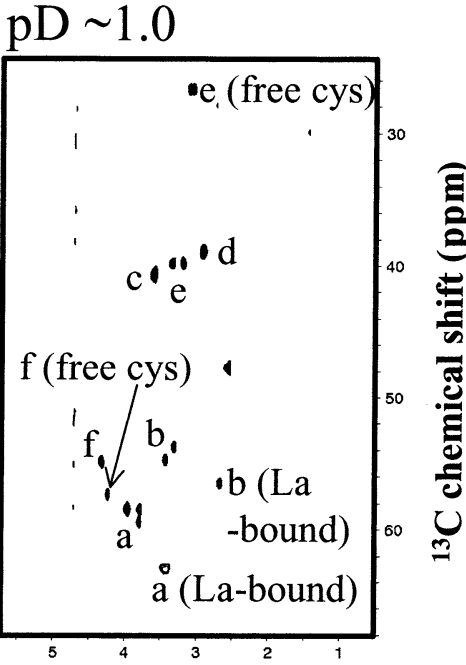

${ }^{1} \mathrm{H}$ chemical shift (ppm)

(c)

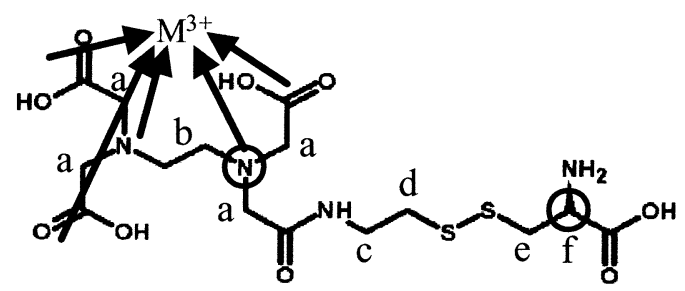

Figure 3. (a) Two-dimensional ${ }^{1} \mathrm{H},{ }^{13} \mathrm{C}-\mathrm{HSQC}$ spectra of L-cysteine connected with MTS-EDTA through the disulfide bond (L-Cys-EDTA) in the presence of $\mathrm{La}^{3+}$ at $\mathrm{pD} 7.5$ (a) and 1.0 (b). In (b), the peaks derived from free cysteine, which did not react with MTS-EDTA, and from the La-bound form (L-Cys-EDTA-La) are also observed. The labels by the sides of peaks on the spectra correspond to those in the chemical formula displayed in (c) on the basis of chemical shift assignments. Possible coordination bonds of the EDTA part to a metal ion $\left(\mathrm{M}^{3+}\right)$ are represented by the arrows on the chemical formula. There are two chiral centers indicated by circles. One is the $\mathrm{C}_{\alpha}$ atom in L-cysteine and the other is the $\mathrm{N}$ atom in the EDTA part closer to the linker, which becomes a chiral center as long as the $\mathrm{N}$ atom entertains a coordinate bond to the metal. Therefore, four cross peaks are observed for the protons on $C_{e}$ and two for $C_{d}$ at $\mathrm{pD} 7.5$, while only two and one cross peaks are observed at $\mathrm{pD} 1.0$. The $\mathrm{pD}$ value of the solution was adjusted to 7.5 by adding a small amount of NaOD. Both spectra were acquired at $303 \mathrm{~K}$ at the proton resonance frequency of $400 \mathrm{MHz}$ with a pulse sequence containing gradient-echo to select the signals passing through the naturally abundant ${ }^{13} \mathrm{C}$ spins.

\section{HSQC spectra of TF-S100C-EDTA (2) and TF-SIOOC-EDTA-CA (4 and 1-epi-4) with metal ions}

The La-chelated form of compound 2, TF-S100CEDTA-La, exhibited a normal ${ }^{15} \mathrm{~N},{ }^{1} \mathrm{H}$ HSQC spectrum (Figure 2a), which could be used as a reference. However, the Dy-bound form, TF-S100C-EDTA-Dy, showed duplicated correlation peaks in 11 residues (N32, G33, A36, K48, A52, L59, T60, I61, G62, S63, and N64) (Figure 2b). According to mass spectrometric analysis, the duplication of the peaks did not stem from protein degradation. The population of the two forms was indistinguishable, which made it impossible to assign the peaks to one or the other species. Most peaks of the amide groups that are positioned closer than $15 \AA$ An in space disappeared, owing to paramagnetic relaxation effects by the coordinated Dy ion. Peaks of the groups that are further than $30 \AA$ were singlets. The amide groups with the doublet features were distributed in a wide range of distances (15 to $30 \AA$ ) from the Dy ion. In order to find out about this doubling of the resonances, further experiments were performed.

EDTA-Cys, 5, with $\mathrm{La}^{3+}$ was investigated by a ${ }^{13} \mathrm{C},{ }^{1} \mathrm{H}-\mathrm{HSQC}$ experiment at $\mathrm{pD} 7.5$ as well as at $\mathrm{pD} 1$. At $\mathrm{pD} 7.5$, each carbon is split in two resonances with similar populations. At pD 1, the affinity of the metal to the EDTA is reduced such that there is fast exchange between the two metal coordinated forms and 

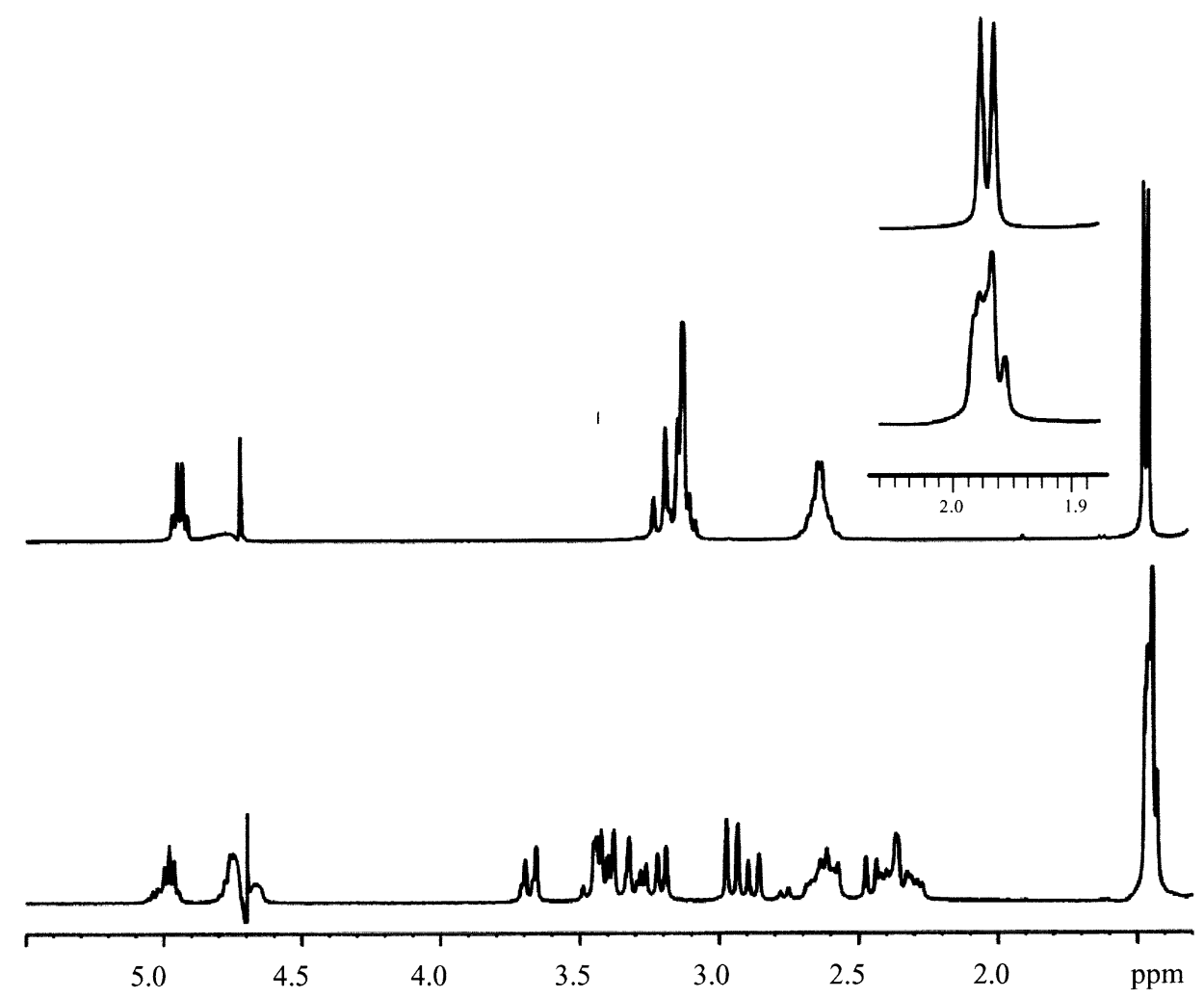

Figure 4. 1D spectrum of compound 6 without (top) and with metal (bottom). Excerpts are shown for the methyl group of the compound. While the peak is a doublet in the absence of metal, the formation of at least two diastereomeric conformations is indicated upon metal chelation.

the non-metal coordinated form. Thus, only one set of signals is observed (Figure 3). This demonstrates the stereoisomerism of the two forms. Since there are, in principle, two stereocenters with the nitrogen pyramidally fixed due to the complexation by the metal and the disulfide bond, we also prepared compound 6 that is lacking the disulfide bridge and recorded a 1D ${ }^{1} \mathrm{H}$ NMR spectrum in the presence of La and observed the same doubling of the resonances (Figure 4). Thus, it is conclusively shown that the EDTA tag forms two sufficiently long lived enantiomeric complexes due to the pyramidally locked nitrogen upon lanthanide complexation. The two sets of signals could not be unambiguously assigned to either of the two diastereomers. In addition, the dipolar couplings were found to be smaller than $1.5 \mathrm{~Hz}$ at $800 \mathrm{MHz}$ which is only three times as large as the error on the coupling constants and rules out the paramagnetic tag $\mathbf{1}$ for general usage. We had started on compound 1 because we had seen multiplication of signals in DTPA analogs that could be brought to coalescence at $80^{\circ} \mathrm{C}$. Due to the much weaker binding of EDTA to lanthanides than
DTPA, we had erroneously expected that the kinetics of interconversion would also speed up. Unfortunately, this turned out to be wrong. It should be also noted that we observed selective line broadening of glutamate residues for trigger factor tagged with $\mathbf{1}$ that were too far away from the tagged site to be induced by the long range effects. We conclude that there is also some non-specific binding of lanthanides to these residues.

We therefore moved on to the interpretation of the spectra of compound $\mathbf{4}$ and 1-epi-4 that are derived from tag 3 and 1-epi-3. Dipolar couplings for compound $\mathbf{4}$ and 1-epi- $\mathbf{4}$ were found ranging between +5 and $-5 \mathrm{~Hz}$ (peak 1) and 8 and $-8 \mathrm{~Hz}$ (peak 2), respectively. Duplication of the signals was not observed for either of the two paramagnetic compounds nor were there effects from unspecific binding of the lanthanide, e.g., to glutamate residues. Anisotropic magnetic susceptibility tensors for 4 and
1-epi-4

Both HSQC spectra of the two diastereomeric complexes could be analyzed in a straightforward manner 
and 51 couplings $(61 \%)$ could be measured for the peak-1 derived compound and 40 (48\%) for the peak-2 derived compound. The assignment was accomplished using the fact that the pseudocontact shifts induced for ${ }^{15} \mathrm{~N}$ and ${ }^{1} \mathrm{H}$ are almost identical in units of ppm. The position of the tag was not optimized to obtain the maximum of dipolar couplings. It can be expected that for larger proteins the percentage of peaks from which dipolar couplings are extracted will increase. For the first compound, we obtained $D_{\mathrm{xx}}=0.10 \mathrm{~Hz}$, $D_{\mathrm{yy}}=7.46 \mathrm{~Hz}$ and $D_{\mathrm{zz}}=-7.56 \mathrm{~Hz}$ and for the second $D_{\mathrm{xx}}=-1.89 \mathrm{~Hz}, D_{\mathrm{yy}}=-6.80 \mathrm{~Hz}$ and $D_{\mathrm{zz}}=8.68 \mathrm{~Hz}$. With the formula for the relationship between dipolar couplings and the anisotropy of the magnetic susceptibility tensor expressed in the eigen frame of the susceptibility tensor

$$
\begin{aligned}
& \begin{aligned}
D_{N H}= & \frac{h B_{0}^{2} \gamma_{H} \gamma_{N}}{4 r_{N H}^{3} 60 k T \pi^{3}}\left(\Delta \chi_{a x}\left(3 \cos ^{2} \theta-1\right)\right. \\
& \left.+\frac{3}{2} \Delta \chi_{r h} \sin ^{2} \theta \cos 2 \phi\right)
\end{aligned} \\
& \text { with } \Delta \chi_{a x}=\frac{2 \chi_{z z}-\chi_{x x}-\chi_{y y}}{2} \\
& \text { and } \Delta \chi_{r h}=\chi_{x x}-\chi_{y y}
\end{aligned}
$$

$$
\begin{aligned}
D_{N H}= & \frac{D_{x x} x_{N H}^{2}+D_{y y} y_{N H}^{2}+D_{z z} z_{N H}^{2}}{r_{N H}^{2}} \\
= & \frac{h B_{0}^{2} \gamma_{H} \gamma_{N}}{r_{N H}^{5} 80 k T \pi^{3}}\left(\chi_{x x} x_{N H}^{2}+\chi_{y y} y_{N H}^{2}\right. \\
& \left.+\chi_{z z} z_{N H}^{2}\right)
\end{aligned}
$$

with $x_{N H}, y_{N H}, z_{N H}$ being the Cartesian coordinates of the ${ }^{15} \mathrm{~N},{ }^{1} \mathrm{H}$-vector under investigation, we arrived at the following values for the anisotropic part of the susceptibility tensor:

Peak 1: $\chi_{x x}=-0.067 * 10^{-32} \mathrm{~m}^{3}, \chi_{y y}=-4.9 *$ $10^{-32} \mathrm{~m}^{3}, \chi_{z z}=5.04 * 10^{-32} \mathrm{~m}^{3}$

Peak 2: $\chi_{x x}=1.26 * 10^{-32} \mathrm{~m}^{3}, \chi_{y y}=4.53 * 10^{-32} \mathrm{~m}^{3}$, $\chi_{z z}=-5.79 * 10^{-32} \mathrm{~m}^{3}$.

Pseudo-contact-shift (PCS) values were calculated for each amide group of compound $\mathbf{4}$, as well as 1-epi4, using the anisotropic magnetic susceptibility tensors as derived from the dipolar couplings.

The pseudocontact shifts were interpreted with Equation 2 where $r_{e H}$ is the distance between the observed proton and the Lanthanide.

$$
\begin{aligned}
\delta_{H}^{p c s}= & \frac{1}{r_{e H}^{3} 12 \pi}\left(\Delta \chi_{a x}\left(3 \cos ^{2} \theta-1\right)+\right. \\
& \left.\frac{3}{2} \Delta \chi_{r h} \sin ^{2} \theta \cos 2 \phi\right),
\end{aligned}
$$

which can be rewritten as:

$$
\delta_{H}^{p c s}=\frac{1}{r_{e H}^{5} 4 \pi}\left(\chi_{x x} x_{N H}^{2}+\chi_{y y} y_{N H}^{2}+\chi_{z z} z_{N H}^{2}\right) .
$$

A fitting program was used to position the paramagnetic ion with respect to the protein. The positions found for peak 1 and peak 2 tagged protein are depicted in Figure 5. As one can see, not only is the position of the metal different in the two diastereomers but also the tensor has rotated considerably. The amount of linear independence can be calculated, by the scalar product between the two Saupe matrices, to be 0.67 , amounting to a projection angle between the two tensors in the five dimensional space of the Saupe matrices of $48^{\circ}$ (Sass et al., 1999).

Despite the fact that for peak 2 tagged compound the maximum coupling should be below $9 \mathrm{~Hz}$, we observed for residue 96 an $\mathrm{NH}$ coupling constant of $10.5 \mathrm{~Hz}$ which will be discussed below.

\section{Discussion}

The EDTA-tag 1 exhibited two problems: First, the dipolar couplings observed were quite small and, second, there was a doubling of resonances. The second problem could be traced back to the occurrence of diastereomeric lanthanide complexes due to the pyramidalisation of the nitrogen in the complex. This could be proven by compound $\mathbf{6}$, which has a stable stereocenter in the $\alpha$-Methylbenzylamide moiety and no further stereocenter. When lanthanide was added, two sets of signals were generated. Since diastereomers differ in their properties, it is not surprising that two susceptibility tensors are observed for $\mathbf{2}$ and consequently two sets of resonances due to different pseudocontact shifts.

In analyzing the origin of the duplication, we excluded protein degradation as well as protein dynamics. Trigger factor is known to interconvert on a fast time scale between an open and closed form (Vogtherr et al., 2002). However, the same duplication of signals was observed when the peptide (SucAla-Ala-Pro-Phe- $\mathrm{pNO}_{2}$ ), a substrate for the cis-trans peptidyl-prolyl isomerase trigger factor, was added. 

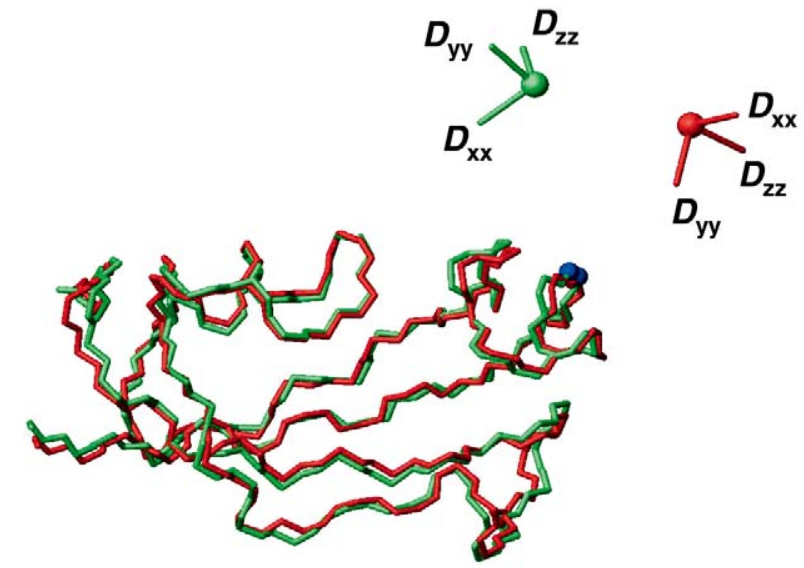

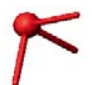

Figure 5. Stereoviews of the calculated structures of the trigger factor using the dipolar couplings measured for compounds $\mathbf{4}$ and 1 -epi-4. The positions of the metal are indicated together with the axes of the susceptibility tensor, as back calculated from the dipolar couplings.

The other problem mentioned is the very small dipolar couplings observed for EDTA-TF-S100C (2), not larger than $1.5 \mathrm{~Hz}$. It was therefore desirable to reduce the number of flexible bonds in the linker and this led us to use the compound $\mathbf{3}$ for tagging. Indeed, for the EDTA-carbonic-acid-TF-S100C, 4, the dipolar couplings were large enough to be measured reliably. The dipolar couplings as well as the pseudocontact shifts differed considerably for the two diastereomeric compounds 4 and 1-epi-4. This is not surprising, since, in a conformationally restricted situation, the orientation of the diastereomeric tags will have to be quite different. The tag works reliably, since we have also observed similar dipolar couplings for attaching the tag to ubiquitin (unpublished results).

\section{Anisotropy of magnetic susceptibility expected from EDTA derivatives}

The size of the anisotropies of the susceptibility tensors found for the two diastereomers can be compared to the tensors known from the literature. We compare with EDTA indole complexes (Kemple et al., 1988) as well as with the recently published Calbindin D9k tensors with different lanthanides (Bertini et al., 2001a) (Table 1).

The ratios of the rhombic to axial components of the tensors are comparable for the EDTA derived compounds 4 and 1-epi-4 and the indole-EDTA compounds, despite the fact that different lanthanides were used. Compared to the values for calbindin, we observed a reduction in the magnitude by a factor of 4 , which indicates a rather rigid tag conformation. If we assumed that the axial components of the calbindin tensor and the EDTA tensor were the same, we would arrive at an order parameter of 0.25 . Additional experiments will have to be performed to measure the absolute size of the alignment tensor of the tag.

\section{Back calculation of pseudo-contact-shifts and dipolar couplings}

We have back calculated the position of the metal from the pseudocontact shifts using the susceptibility tensor, as derived from the dipolar couplings and a simple fitting program. The position will only be an average, since, from the reduced susceptibility tensor, there is expected to be flexibility of the tag. The position fits quite well with the expected distance between the center of EDTA and the sulfur atom of Cys to which it is attached (Figure 5). The Q-value

$$
Q=\sqrt{\frac{\sum_{i}\left(\delta_{H_{i}}^{p c s, \exp }-\delta_{H_{i}}^{p c s, c a l c}\right)^{2}}{\sum_{i}\left(\delta_{H_{i}}^{p c s, \exp }\right)^{2}}},
$$

as derived from the structures shown in Figure 5 is below 0.2 for both cases, despite the fact that it is expected that the tag moves with respect to the protein.

A structure was calculated using XPLOR-NIH with the two sets of dipolar couplings yielding a structure whose rmsd to the published trigger factor structure is $0.85 \AA$. The correlation of the experimental and back calculated dipolar couplings is shown in the supplementary material.

Another interesting observation is made for the trigger factor that is tagged with peak 2 . Here, we find a dipolar N,H-coupling for residue 96 of $10.5 \mathrm{~Hz}$ that 
Table 1. Comparison of the magnetic susceptibility tensors for lanthanide loaded indole-EDTA, calbindin D9k and compounds 4 and 1-epi-4

\begin{tabular}{lcccc}
\hline$\Delta \chi_{a x} ; \Delta \chi_{r h}$ both in $10^{-32} \mathrm{~m}^{3}$ & Indole EDTA & Calbindin & $\mathbf{4}$ & 1-epi-4 \\
\hline $\mathrm{Dy}$ & & $34.7 ; 20.3$ & $7.56 ; 4.83$ & $-8.68 ;-3.27$ \\
$\mathrm{Pr}$ & $-3.46 ;-1.63$ & $3.4 ; 2.11$ & & \\
$\mathrm{Nd}$ & $-2.35 ;-1.47$ & $1.74 ; 0.46$ & \\
$\mathrm{Eu}$ & $-3.49 ;-0.22$ & $-2.34 ;-1.63$ & \\
\hline
\end{tabular}

is not possible with the alignment tensor calculated. This could indicate that the protein part that is tagged moves with respect to the remainder of the protein.

\section{Conclusion}

We have synthesized MTS-EDTA and MTS-EDTAcarbonic-acid, which can be connected to a cysteine residue through the disulfide bond and can coordinate a lanthanide ion. Both compounds fulfill the following conditions: (a) high metal binding constants, (b) maintenance of the structures (biological activities) of attached proteins, (c) water solubility, (d) high selectivity towards one specific site of the protein, (e) high quantitative yield of the tagging reaction, (f) long term stability, and (g) simple purification procedures after the tagging reaction. While stereochemical complications finally rendered the MTS-EDTA tag not useful, the MTS-EDTA-carbonic-acid tag provides dipolar couplings in the range of $\pm 5 \mathrm{~Hz}$ or $\pm 8 \mathrm{~Hz}$ at $800 \mathrm{MHz}$ as well as pseudocontact shifts of up to $0.5 \mathrm{ppm}$. The dipolar couplings and the pseudocontact shifts could be used for structure refinement and the position of the lanthanide could be obtained. Two diastereomeric forms induce alignment tensors that are linearly independent of each other to an extent that each of them could be used for studies of dynamics as have been introduced with external alignment media (Meiler et al., 2001; Peti et al., 2002).

A further application of such tags lies in the possibility to study transferred dipolar couplings in systems containing the mixture of free and complex forms between a protein and the related ligand in a pure way, since there will be no dipolar coupling induced in a ligand unless it is bound to the tagged protein.

A further benefit of using paramagnetic alignment comes from the fact that, in addition to orientational information, distance information can also be ob- tained, e.g., of ligands bound to paramagnetically tagged proteins.

\section{Acknowledgements}

This work was supported by the DFG (Gr1112/2-4, 10-1), the MPG, the Fonds der Chemischen Industrie, the Humboldt-Foundation and the Training Network RTN HPRN CT 200000092 (L.V.), and the Postdoctoral Fellowships for Research Abroad from Japan Society for the Promotion of Science (T.I.). Prof Harald Schwalbe and Dr Ariane Tüchelmann, both at Frankfurt University at that time, were involved in early stages of this work. Mrs Brigitte Voigt helped with the synthesis. Fruitful discussions with Prof Ivano Bertini and Prof Claudio Luchinat, Florence University, on paramagnetic tagging are gratefully acknowledged. Fruitful discussions with Dr Andrei Leonov are also gratefully acknowledged. The authors wish to thank Mrs Kerstin Overkamp, Mrs Ellen Lichte and Mrs Elke Stirnal for the HPLC purification and Kerstin Overkamp also for mass spectrometric analyses by ESI-MS. We gratefully acknowledge measurement of MALDI MS spectra by Dr Henning Urlaub. Finally we would like to thank Dr Colan Hughes for carefully reading the manuscript.

\section{References}

Arya, R. and Garyepy, J. (1991) Bioconjugate Chem., 2, 323-326. Battiste, J.L. and Wagner, G. (2000) Biochemistry, 39, 5355-5365. Beger, R.D., Marathias, V.M., Volkman, B.F. and Bolton, P.H. (1998) J. Magn. Res., 135, 256-259.

Bertini, I., Janik, M.B.L., Lee, Y.-M., Luchinat, C. and Rosato, A. (2001a) J. Am. Chem. Soc., 123, 4181-4188.

Bertini, I., Luchinat, C. and Parigi, G. (2001b) Solution NMR of Paramagnetic Molecules - Application to metallobiomolecules and models, Current Methods in Inorganic Chemistry, Vol. 2, Elsevier Science B.V., Amsterdam.

Bertini, I., Luchinat, C. and Piccioli, M. (2001c) Paramagnetic probes in metalloproteins. In Methods in Enzymology, Vol. 339, 
James, T.L., Doetsch, V. and Schmitz, U. (Eds.), Academic Press, San Diego, pp. 314-340.

Bertini, I., Donaire, A., Jimenez, B., Luchinat, C., Parigi, G., Piccioli, M. and Poggi, L. (2001d) J. Biomol. NMR, 21, 85-98.

Briggman, K.B. and Tolman, J.R. (2003) J. Am. Chem. Soc., 125, 10164-10165.

Burns, P.D. and LaMar, G.N. (1982) J. Magn. Reson., 46, 61-68.

Clore, G.M. and Gronenborn, A.M. (1998) PNAS 95, 5891-5898.

Contreras, M.A., Ubach, J., Millet, O., Rizo, J. and Pons, M. (1999) J. Am. Chem. Soc., 121, 8947-8948.

Datwyler, S.A. and Meares, C.F. (2000) Trends Biochem. Sci., 25, 408-414.

Donaldson, L.W., Skrynnikov, N.R., Choy, W.Y., Muhandiram, D.R., Sarkar, B., Forman-Kay, J.D. and Kay, L.E. (2001) J. Am. Chem. Soc., 123, 9843-9847.

Dvoretsky, A., Gaponenko, V. and Rosevear, P.R. (2002) FEBS Lett., 528, 189-192.

Ebright, Y.W., Chen, Y., Pendergrast, S. and Ebright, R.H. (1992) Biochemistry, 31, 10664-10670.

Ermacora, M.R., Delfino, J.M., Cuenoud, B., Schepartz, A. and Fox, R.O. (1992) Proc. Natl. Acad. Sci. U.S.A., 89, 6383-6387.

Feeney, J., Birdsall, B., Bradbury, A.F., Biekofsky, R.R. and Bayley, P.M. (2001) J. Biomol. NMR, 21, 41-48.

Gaponenko, V., Altieri, A.S., Li, J. and Byrd, R.A. (2002) J. Biomol. NMR, 24, 143-148.

Gaponenko, V., Dvoretsky, A., Walsby, C., Hoffman, B.M. and Rosevear, P.R. (2000) Biochemistry, 39, 15217-15224.
Gochin, M. (2002) Struct. Folding Des., 8, 441-452.

Kemple, M.D., Ray, B.D., Lipkowitz, K.B., Prendergast, F.G. and Rao, B.D.N. (1988) J. Am. Chem. Soc., 110, 8275-8287.

Hus, J.-C., Peti, W., Griesinger, C. and Brüschweiler, R. (2003) J. Am. Chem. Soc., 125, 5596-5597.

Ma, C. and Opella, S.J. (2000) J. Magn. Reson., 146, 381-384.

Meiler, J., Peti, W. and Griesinger, C. (2003) J. Am. Chem. Soc., 125, 8072-8073.

Meiler, J., Prompers, J., Peti, W., Griesinger, C. and Brüschweiler, R. (2001) J. Am. Chem. Soc., 123, 6098-6107.

Peti, W., Meiler, J., Brüschweiler, R. and Griesinger, C. (2002) J. Am. Chem. Soc., 124, 5822-5833.

Prosser, R.S., Volkov, V.B. and Shiyanovskaya, I.V. (1998) Biophys. J., 75, 2163-2169.

Sass, J., Cordier, F., Hoffman, A., Rogowski, M., Cousin, A., Omichinski, J.G., Löwen, H. and Grzesiek, S. (1999) J. Am. Chem. Soc., 121, 2047-2055.

Tjandra, N. and Bax, A. (1997) Science, 278, 1111-1114.

Tolman, J.R. (2002) J. Am. Chem. Soc., 124, 12020-12030.

Tolman, J.R., Flanagan, J.M., Kennedy, M.A. and Prestegard, J.H. (1995) Proc. Natl. Acad. Sci., 92, 9279-9283.

Vogtherr, M., Jacobs, D.M., Parac, T.N., Maurer, M., Pahl, A., Saxena, K., Rueterjans, H., Griesinger, C. and Fiebig, K.M. (2002) J. Mol. Biol., 318, 1097-1115.

Weidner, J.P. and Block, S.S. (1972) J. Med. Chem., 15, 564-567.

Woehnert, J., Franz, K.J., Nitz, M., Imperiali, B. and Schwalbe, H. (2003) J. Am. Chem. Soc., 125, 13338-13339. 